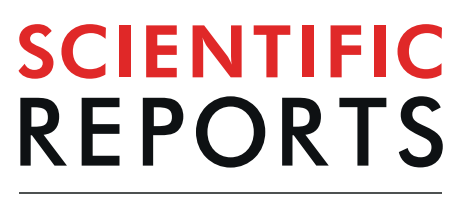

natureresearch

Check for updates

\title{
Efficient uptake and retention of iron oxide-based nanoparticles in HeLa cells leads to an effective intracellular delivery of doxorubicin
}

R. C. Popescu, ${ }^{1,2,3}$, D. Savu ${ }^{1 凶}$, I. Dorobantu' ${ }^{1}$, B. S. Vasile², H. Hosser ${ }^{4}$, A. Boldeiu $^{5}$, M. Temelie $^{1}$, M. Straticiuc ${ }^{6}$, D. A. Iancu ${ }^{6}$, E. Andronescu ${ }^{2}$, F. Wenz ${ }^{7}$, F. A. Giordano ${ }^{3}$, C. Herskind ${ }^{3}$ \& M. R. Veldwijk ${ }^{3 凶}$

The purpose of this study was to construct and characterize iron oxide nanoparticles (IONP $\left.\mathrm{CO}_{\mathrm{CO}}\right)$ for

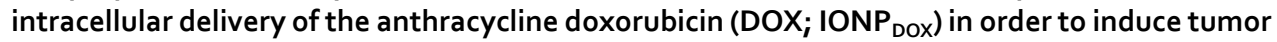
cell inactivation. More than $80 \%$ of the loaded drug was released from IONP $\mathrm{P}_{\text {Dox }}$ within $24 \mathrm{~h}(100 \%$ at $70 \mathrm{~h}$ ). Efficient internalization of $I O N P_{\text {Dox }}$ and $I O N P_{C O}$ in HeLa cells occurred through pino- and endocytosis, with both IONP accumulating in a perinuclear pattern. IONP ${ }_{c o}$ were biocompatible with maximum $27.9 \% \pm 6.1 \%$ reduction in proliferation $96 \mathrm{~h}$ after treatment with up to $200 \mu \mathrm{g} / \mathrm{mL}$ $I \mathrm{ONP}_{\mathrm{CO}}$. Treatment with IONP $\mathrm{DOx}_{\mathrm{DOx}}$ resulted in a concentration- and time-dependent decrease in cell proliferation $\left(\mathrm{IC}_{50}=27.5 \pm 12.0 \mu \mathrm{g} / \mathrm{mL}\right.$ after $\left.96 \mathrm{~h}\right)$ and a reduced clonogenic survival (surviving fraction, $\mathrm{SF}=0.56 \pm 0.14$; versus $\mathrm{IONP}_{\mathrm{CO}}(\mathrm{SF}=1.07 \pm 0.38)$ ). Both IONP constructs were efficiently internalized and retained in the cells, and IONP ${ }_{\mathrm{DOx}}$ efficiently delivered $\mathrm{DOX}$ resulting in increased cell death vs IONP $_{\text {CO. }}$.

Chemotherapy is an essential systemic component in modern multimodal cancer treatment, yet one of the main disadvantages of anticancer chemotherapeutics is toxicity to the normal tissue.

The use of nano-sized carriers as intracellular transporters for the active substances not only promises to reduce the total drug amount administered, while potentially improving the treatment's efficiency by enhancing the local dose in the tumour, but also can help to improve the specificity and targeting of the active substance, thereby reducing the side-effects associated with chemotherapy ${ }^{1}$.

In nano-carriers, drugs can be transported to the tumour site through the enhanced permeability and retention effect ${ }^{2-4}$, magnetic targeting ${ }^{5-8}$ and protected until they find a triggering stimuli to release, like $\mathrm{pH}$ variations $^{9-12}$, temperature ${ }^{13,14}$, radiation-induced release ${ }^{15-19}$.

The use of iron oxide nanoparticles in the construction of nano-systems for the delivery of chemotherapeutics not only enables active magnetic targeting to the tumour site, but also offers additional functions that make them suitable for diagnosis (contrast substance in MRI ${ }^{20-22}$ ) or enhanced anticancer activity using hyperthermia ${ }^{23}$.

Conjugation with other compounds can add to the multi-functionality of these nanomaterials and implement properties such as increased and/or specific ${ }^{24-26}$ internalization in cancer cells, but can also help to modulate the

1"Horia Hulubei" National Institute for Research and Development in Physics and Nuclear Engineering, Department of Life and Environmental Physics, Reactorului 30, 077125, Magurele, Romania. ${ }^{2}$ Politehnica University of Bucharest, Department of Science and Engineering of Oxide Materials and Nanomaterials, Polizu 1-7, 011061, Bucharest, Romania. ${ }^{3}$ Heidelberg University, Medical Faculty Mannheim, Universitätsmedizin Mannheim, Department of Radiation Oncology, Theodor-Kutzer-Ufer 1-3, 68167, Mannheim, Germany. ${ }^{4}$ Heidelberg University, Medical Faculty Mannheim, Universitätsmedizin Mannheim, Center for Biomedicine and Medical Technology, Department of Anatomy and Developmental Biology, Theodor-Kutzer-Ufer 1-3, 68167, Mannheim, Germany. ${ }^{5}$ National Institute for Research and Development in Microtechnologies, Laboratory of Nanobiotechnology, Erou lancu Nicolae 12A, 077190, Bucharest, Romania. "'Horia Hulubei" National Institute for Research and Development in Physics and Nuclear Engineering, Department of Applied Nuclear Physics, Reactorului 30, 077125, Magurele, Romania. ${ }^{7}$ University Medical Center Freiburg, Hugstetter Straße 55, 79106, Freiburg, Germany. ${ }^{凶}$-mail: dsavu@nipne.ro; Marlon.Veldwijk@medma.uni-heidelberg.de 

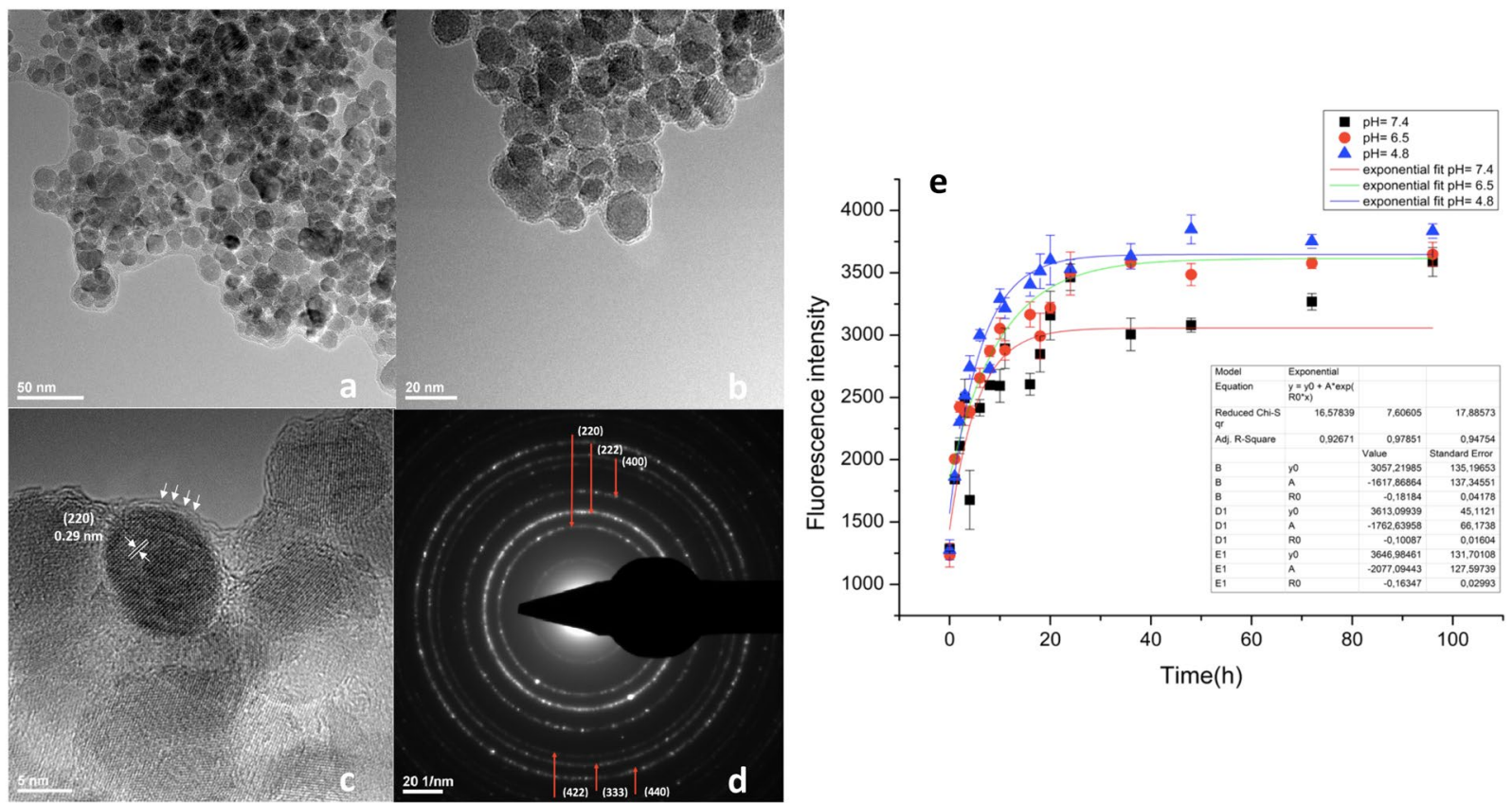

Figure 1. Structural and compositional characterization of IONP. (a,b) Transmission electron microscopy (TEM); (c) High resolution (HR)- TEM; (d) Selected area electron diffraction spectrum (SAED); (E) Delivery of doxorubicin from the $\mathrm{IONP}_{\mathrm{DOX}}$ construct at $37^{\circ} \mathrm{C}(0-96 \mathrm{~h})$.

release of the active substance: (1) by protecting the drug, (2) by delaying the release or (3) by releasing the substance on demand (mediated by $\mathrm{pH}$, heat, light, biological enzymes, etc.).

For these reasons, we designed and synthesized core-shell iron oxide $\left(\mathrm{Fe}_{3} \mathrm{O}_{4}\right)$ nanoparticles functionalized with soft polyethylene glycol $(\mathrm{PEG})$ layers $\left(\mathrm{IONP}_{\mathrm{CO}}\right)$ that entrap and protect the drug, the anthracycline doxorubicin (DOX) until delivery. DOX was chosen as a chemotherapeutic model due to its current clinical use in the management of various cancers, including cervical cancer, but also because of its traceability (fluorescence upon excitation). The novelty of this strategy comes from the synthesis method which is based on a room temperature adapted Massart approach combined with a post-synthesis encapsulation, resulting in core-shell- like PEG-conjugated highly crystalline $\mathrm{Fe}_{3} \mathrm{O}_{4}$ nanoparticles.

The aim of the study was to determine the uptake and retention efficacy of the IONP and to test their ability to induce cell death in HeLa cells by incorporation of DOX into the $\mathrm{IONP}_{\mathrm{CO}}$. To the best of our knowledge, we evaluated for the first time the intracellular retaining and fate of PEG-coated iron oxide nanoparticles (qualitatively and also quantitatively) over a time range longer than one complete cell cycle, after the NP exposure had been discontinued. IONP $\mathrm{CO}$ had the ability to encapsulate and deliver the chemotherapeutic doxorubicin directly into the intracellular cytoplasmic compartment, but were also retained until the death of the malignant cell.

\section{Results}

Physical and chemical characterization of the IONP. Synthesis of IONP using a three-step synthesis method leads to the generation of highly crystalline individual iron oxide cores with average diameters of $12.82 \pm 2.73 \mathrm{~nm}$ (Fig. 1a-c), showing diffraction rings characteristic for face-centred spinel structured magnetite ((220), (222), (400), (422), (333), (440)) (Fig. 1d). Individually covered NPs with PEG were organized as coreshell-like nano-constructs, as shown by high resolution transmission electron microscopy (HR-TEM; Fig. 1c; PEG shell emphasized with white arrows). HR-TEM confirmed the data on crystallinity and emphasized the presence of the (220) plane of $0.29 \mathrm{~nm}$ width (Fig. 1c), characteristic for the magnetite phase. Conjugation with PEG also lead to a stabile dispersion of the NPs stock solution in water, a mean hydrodynamic diameter of $164.2 \mathrm{~nm}$ being measured (prior to ultrasound dispersion). Zeta potential measurements showed good stability ( $14.80 \mathrm{mV}$ for stock solutions with no prior ultrasound treatment). Loading of the DOX resulted in an increase in the hydrodynamic diameter ( $369.1 \mathrm{~nm}$ mean diameter) and a change of surface charge to negative values $(-20.9 \mathrm{mV}$ zeta potential). Both of the constructs were monodisperse systems, as the values of the polydispersity index (PDI) were below 0.3 ( 0.233 for $\mathrm{IONP}_{\mathrm{CO}}$ and 0.238 for $\left.\mathrm{IONP}_{\mathrm{DOX}}\right)$. Quantitative determination of the DOX-loading content in IONP showed a value of $1.11 \mathrm{wt} \%$ (Supplementary Material Section 2).

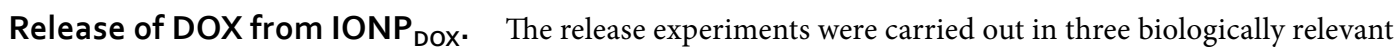
culture media with different $\mathrm{pH}$ values: $7.4 \mathrm{pH}$, which is characteristic for physiologic plasma, $6.5 \mathrm{pH}$ which is relevant for tumour microenvironment ${ }^{27,28}$ and $4.8 \mathrm{pH}$, which is encountered in the intra-lysosomal compartment ${ }^{29}$. $\mathrm{IONP}_{\mathrm{DOX}}$ showed a rapid, initial release which was not significantly affected by the $\mathrm{pH}$ (Fig. 1e).

Hemocompatibility of IONP. The hemolytic potential (Supplementary Table S2) was below 5\% (ASTM standard E2524-08). Thus, neither IONP $\mathrm{CO}_{\text {O }}$ nor IONP $\mathrm{DOx}_{\text {ox }}$ produced a hemolytic effect at the concentrations used. 


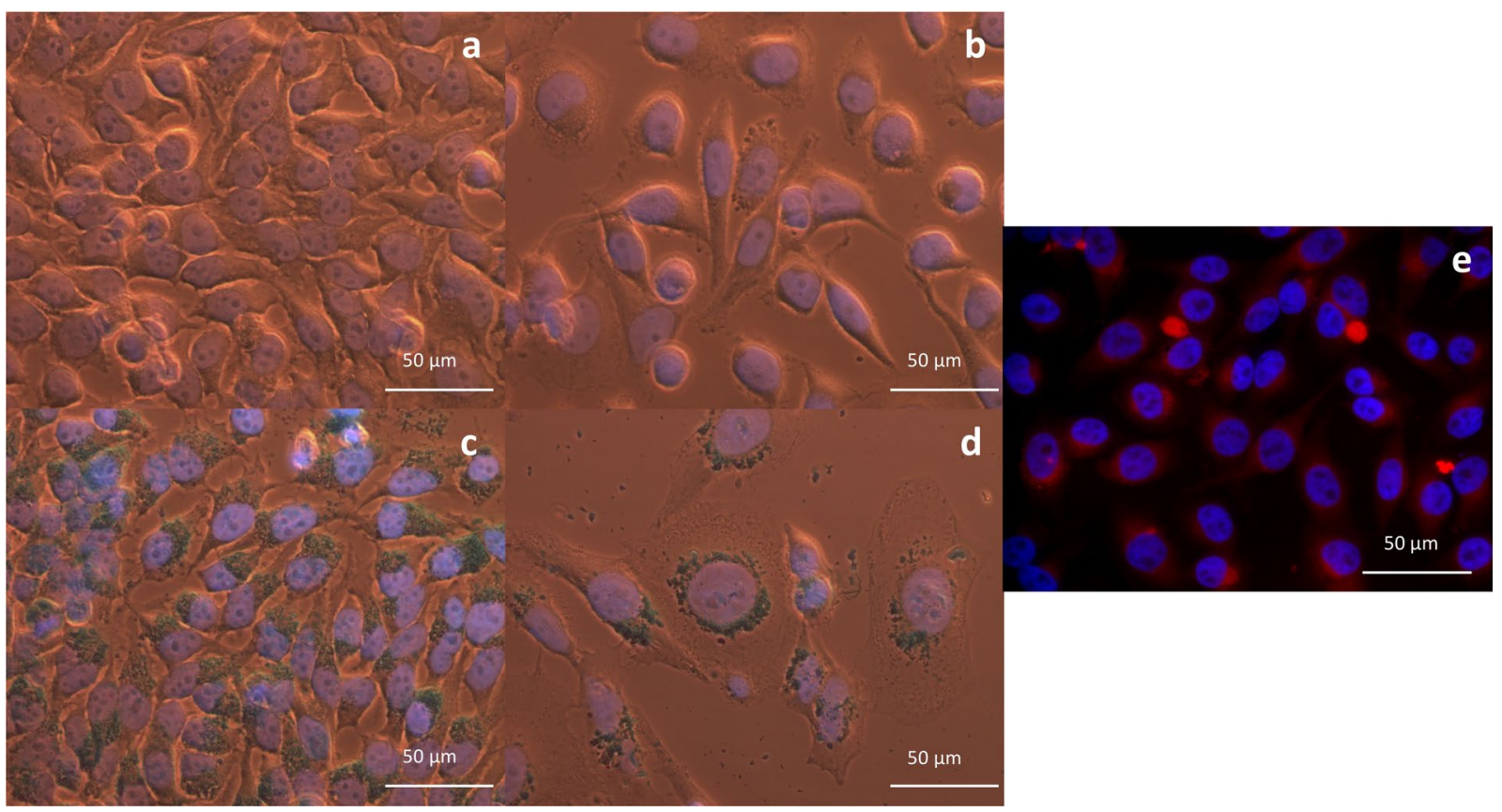

Figure 2. Morphological characterization of HeLa cells after IONP treatment. Bright-field images of HeLa cells incubated for $16 \mathrm{~h}$ with: (a) PBS; (b) DOX $\left(1.11 \mu \mathrm{g} / \mathrm{mL}\right.$ equivalent concentration to $\mathrm{IONP}_{\mathrm{Dox}}$; Supplementary data Section 2); (c) $\operatorname{IONP}_{\mathrm{CO}}(100 \mu \mathrm{g} / \mathrm{mL})$ and $(\mathbf{d}) \mathrm{IONP}_{\mathrm{DOX}}(100 \mu \mathrm{g} / \mathrm{mL}) ; \mathrm{IONP}$ are stained blue after Prussian Blue staining, 40x magnification (oil). Fluorescence images of HeLa cells $24 \mathrm{~h}$ after incubation for $16 \mathrm{~h}$ with: (e) $100 \mu \mathrm{g} / \mathrm{mL}$ or IONP $_{\text {DOX }}$. Nuclei are stained with DAPI (blue), red staining is fluorescence from incorporated DOX; 40x magnification.

Internalizing and retention of IONP in HeLa cells. At $24 \mathrm{~h}$ after $16 \mathrm{~h}$ incubation with nanoparticles, internalized IONP were shown not to translocate into the nucleus, remaining organized as light blue-coloured agglomerates covering the outer nuclear membrane (Fig. 2c,d). The morphology of the samples was not affected in case of IONP $\mathrm{CO}_{\mathrm{C}}$-exposed HeLa cells (Fig. 2c). However, the cell density was affected by DOX from the IONP $\mathrm{DOX}_{\mathrm{DOX}}$ samples (Fig. 2d) and by equivalent amounts of free DOX (Fig. 2b). The morphology of IONP Dox $_{\text {-treated HeLa }}$ cells changed, becoming rounder and larger. In addition, nuclei increased in volume. By adjusting the focusing plane, different depth levels of accumulated IONP could be differentiated in the cell (Supplementary Figs. S1, S2). Thus, most nanoparticle agglomerates appeared in a perinuclear location in the cell. Still, some can be seen directly interacting with the cell membrane during the internalizing process.

In case of $\mathrm{IONP}_{\mathrm{DOX}}$, localizing of the nano-constructs inside the cells was confirmed by fluorescence detection of DOX $24 \mathrm{~h}$ after $16 \mathrm{~h}$ incubation with DOX-loaded nanoparticles (Fig. 2e). A signature red fluorescence of the $\mathrm{IONP}_{\mathrm{DOX}}$ aggregates (sub-micron spherical structures in the peri-nuclear area and the cytoplasm) was observed with a weaker intensity in the remaining cytoplasm and in the nucleus (Fig. 2e).

Transmission electron images were acquired for HeLa cells exposed to different concentrations of IONP (100 and $500 \mu \mathrm{g} / \mathrm{mL}$ ) for $16 \mathrm{~h}$ in order to show their internalization and localization in HeLa cells at $24 \mathrm{~h}$ after NP removal. This technique was also employed to potentially study the mechanisms of entrapment. Figure 3 emphasized the localizing of IONP as agglomerates in the perinuclear area and the cytoplasm. The results (Fig. 3e-g and Supplementary Fig. S4) show that both types of constructs are internalized via macropinocytosis (Fig. 3e) and sometimes smaller aggregates are internalized via endocytosis (Fig. 3f), eventually being transferred in lysosomes (Fig. 3g). At $24 \mathrm{~h}$ after the end of NP incubation period, the $\mathrm{IONP}_{\mathrm{CO}}$ appeared to be entrapped in intracellular vesicles (Fig. 3a,b and, while $\mathrm{IONP}_{\mathrm{DOX}}$ aggregates were localized in both vesicular structures and appeared free in cytoplasm (Fig. 3c,d).

Particle-induced X-Ray emission (PIXE) quantitative analysis of $\mathrm{Fe}_{3} \mathrm{O}_{4}$ interacting with HeLa cells yielded a concentration of $31.66 \pm 3.06 \mathrm{pg} \mathrm{Fe}_{3} \mathrm{O}_{4}$ /cell in HeLa cells exposed to $\mathrm{IONP}_{\mathrm{CO}}$, and $115.2 \pm 9.8 \mathrm{pg} \mathrm{Fe}_{3} \mathrm{O}_{4} /$ cell for $\operatorname{IONP}_{\text {DOX }}$ (Fig. 3h).

Effect of IONP on the proliferation kinetics of HeLa cells. The effect of IONP on the proliferation of HeLa cells was determined for a broad concentration range of $\operatorname{IONP}_{\mathrm{CO}}$ and $\operatorname{IONP}_{\mathrm{DOX}}(0-200 \mu \mathrm{g} / \mathrm{mL}) \mathrm{during}$ 48-96 h incubation. Results were shown relative to controls (untreated HeLa cells $=100 \%$ ).

With $\mathrm{IONP}_{\mathrm{CO}}$, a time and concentration-dependent decrease in proliferation was observed until $72 \mathrm{~h}$ of incubation with nanoparticles (Fig. 4a,b). All results were statistically significant relative to untreated cells (One-way ANOVA, $\mathrm{p}<0.05)$. However, after $96 \mathrm{~h}$ of incubation, no significant changes in relative absorbance were observed compared to control samples, a maximum reduction of relative absorbance being $10.88 \pm 7.68 \%$ for the highest concentration employed ( $200 \mu \mathrm{g} / \mathrm{mL}$ IONP) (Fig. $4 \mathrm{c})$. 


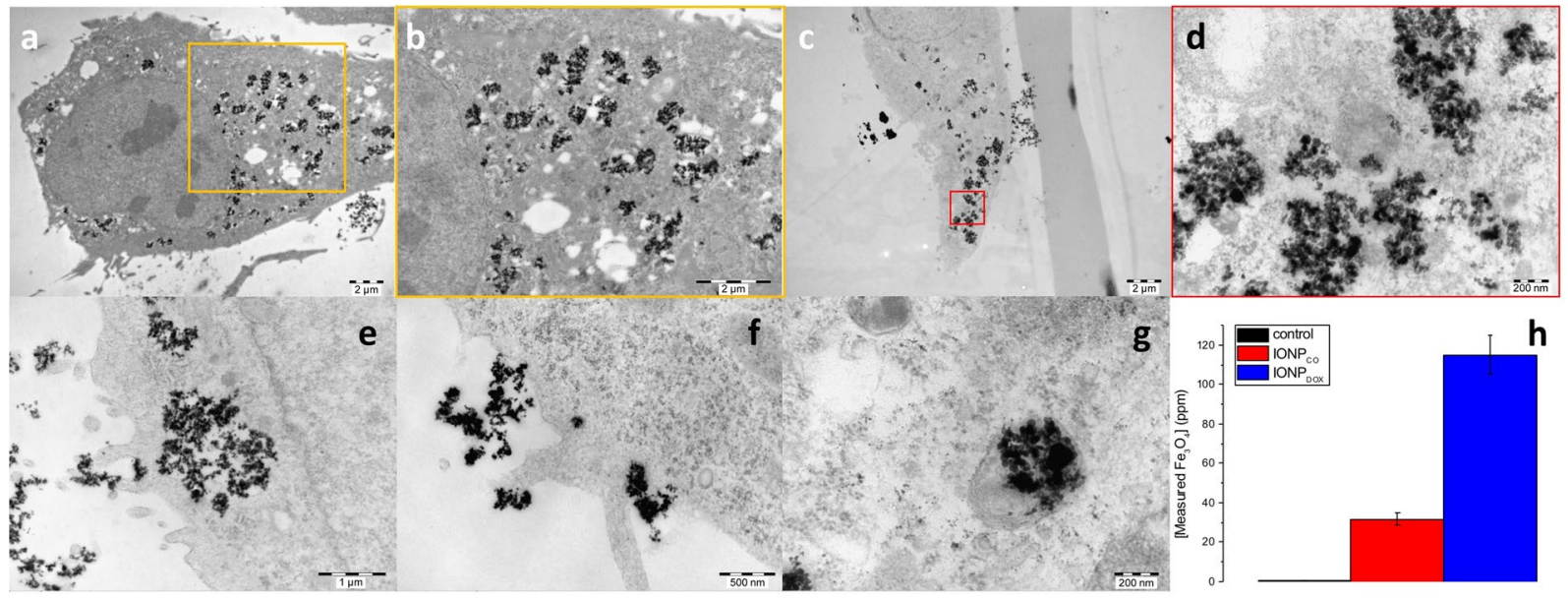

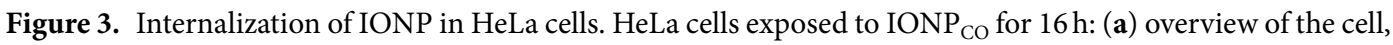
scale $=2 \mu \mathrm{m}$; (b) magnification of the area marked with yellow square in (a); HeLa cells exposed to IONP $\mathrm{DOX}_{\mathrm{D}}$ for 16h: (c) overview of the whole cell, scale $=2 \mu \mathrm{m}$; (d) magnification of the area marked with red square in (c), scale $=200 \mathrm{~nm}$. HeLa cells exposed to IONP for $16 \mathrm{~h}$ : (e) detail of macropinocytosis internalization of IONP, scale $=1 \mu \mathrm{m} ;(\mathbf{f})$ detail of endocytosis internalization of IONP, scale $=500 \mathrm{~nm} ;(\mathbf{g})$ detail of lysosome entrapment of IONP, scale $=200 \mathrm{~nm}$ and (h) Quantity of internalized $\mathrm{Fe}_{3} \mathrm{O}_{4}$ in HeLa cells exposed to 0, respectively $100 \mathrm{ppm}$ IONP during $16 \mathrm{~h}$ at $24 \mathrm{~h}$ after NP removal; data are presented as percentage of untreated control and are shown as mean \pm SEM $(n=3)$.
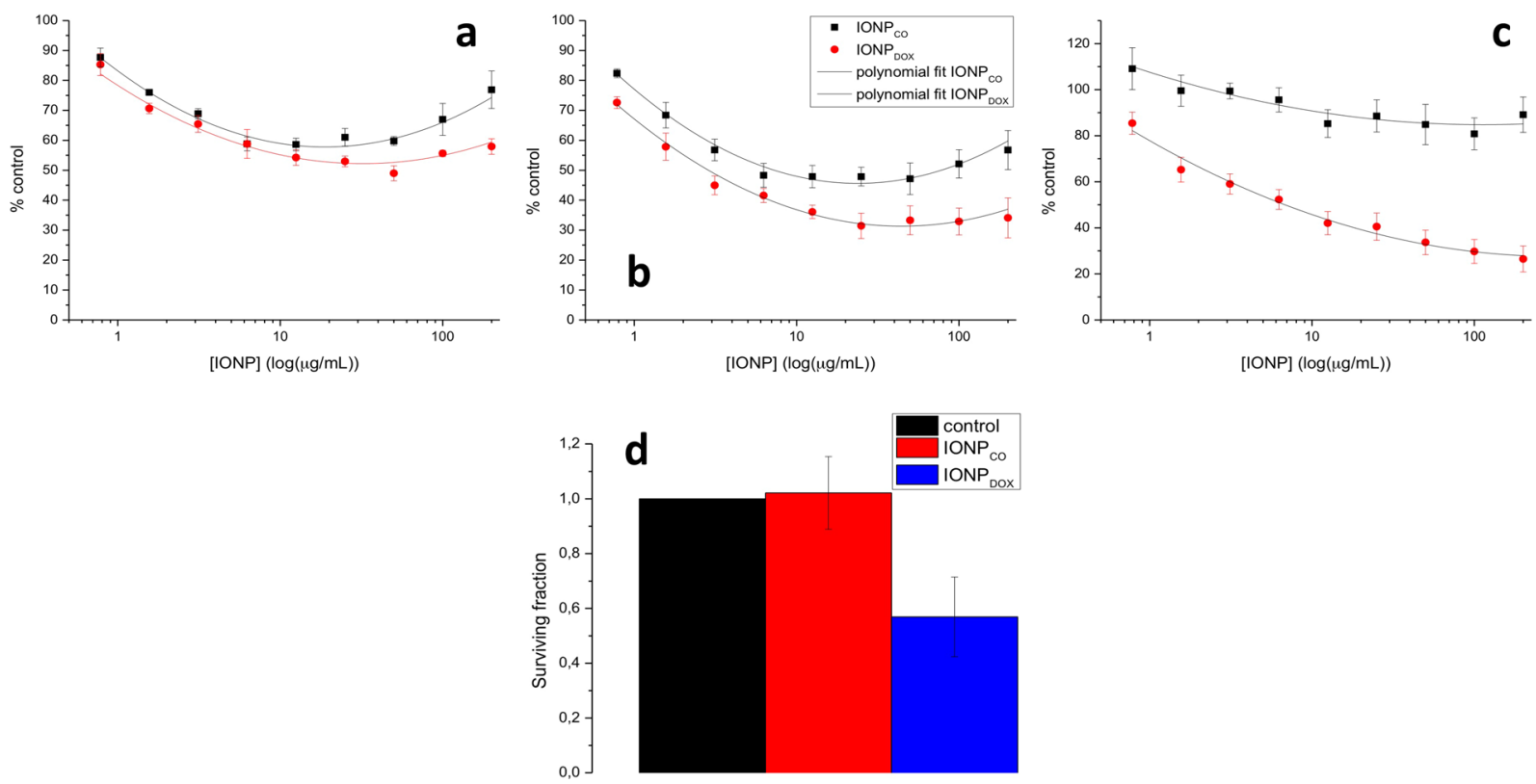

Figure 4. Cytotoxicity of IONP on HeLa cells. (a-c) Proliferation kinetics of HeLa cells incubated with IONP during 48, 72 and 96h. One-way ANOVA statistical analysis revealed a significant difference between treated groups and control; Two-way ANOVA statistical analysis proved significant difference between IONPs and DOX-IONPs $(\mathrm{P}<0.0001$ for $48 \mathrm{~h}$; $\mathrm{P}<0.0001$ for $72 \mathrm{~h}$; $\mathrm{P}<0.0001$ for $96 \mathrm{~h})$. Also, the presence of DOX in the construct induced a significant reduction of proliferation, compared to equivalent concentrations of IONP $\mathrm{CO}_{\mathrm{CO}}$ $(\mathrm{P}=0.0003$ for $48 \mathrm{~h} ; \mathrm{P}<0.0001$ for $72 \mathrm{~h} ; \mathrm{P}<0.0001$ for $96 \mathrm{~h}$ ). (d) Clonogenic survival of HeLa cells seeded in the colony formation assay after exposure to $100 \mu \mathrm{g} / \mathrm{mL}$ IONP for $16 \mathrm{~h}$. Data are presented as percentage of untreated control and are shown as mean $\pm \operatorname{SEM}(\mathrm{n}=3)$.

DOX-free nanoparticles caused a delay in cells proliferation up to $72 \mathrm{~h}$. This effect was diminished at short incubation periods $(24 / 48 \mathrm{~h})$, the effect of $\mathrm{IONP}_{\mathrm{CO}}$ on the HeLa cells could be rather correlated with an initial cytotoxic or growth inhibitory effect, as control cells normally underwent maximum 2 divisions during this time interval (doubling time of HeLa cells is $\sim 18 \mathrm{~h}^{30}$ ). The effect on the cell's ability to reduce the tetrazolium salt might 

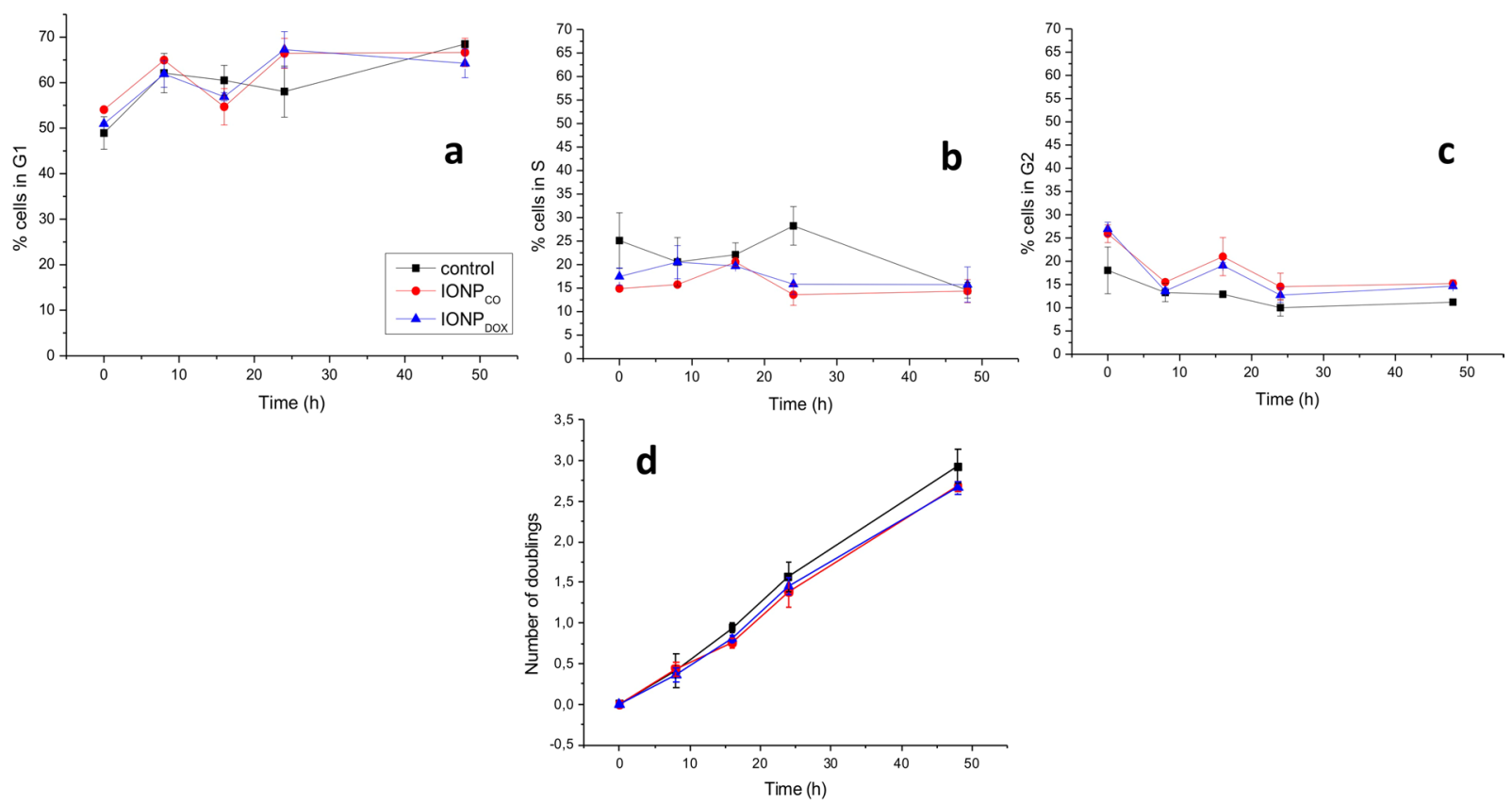

Figure 5. Flow cytometry of HeLa cells after IONP exposure during $16 \mathrm{~h}$. Cell cycle distribution (a-c) and doubling time (d) of HeLa cells incubated with 0 , respectively $100 \mu \mathrm{m}$ IONP during $16 \mathrm{~h}(\mathrm{n}=3)$. Data are presented as percentage of untreated control and are shown as mean $\pm \operatorname{SEM}(n=3)$.

be justified by an initial toxicity with time (Fig. 4a,b). At later time points (96h), showing values close to control cells suggested that the initial effect of the IONP was rather based on growth delay/inhibition than cell kill/cytotoxicity (Fig. 4c).

Incubation of HeLa cells with $\mathrm{IONP}_{\mathrm{DOx}}$ showed a clear cytotoxic effect that increased with the concentration of administered NP and time (Fig. $4 \mathrm{a}-\mathrm{c}$ ). The calculated $\mathrm{IC}_{50}$ values were $27.83 \pm 7.99 \mu \mathrm{g} / \mathrm{ml}$ for $48 \mathrm{~h}$ of NP incubation, $2.31 \pm 0.32 \mu \mathrm{g} / \mathrm{ml}$ for $72 \mathrm{~h}$ of NP incubation, respectively $9.01 \pm 4.68 \mu \mathrm{g} / \mathrm{ml}$ for $96 \mathrm{~h}$ of NP incubation. While IONP $\mathrm{CO}_{\mathrm{CO}}$ showed nearly no effect on the metabolic activity of HeLa cells at $96 \mathrm{~h}$ compared to untreated controls, even at very high doses $(200 \mu \mathrm{g} / \mathrm{ml})$, incubation with $\mathrm{IONP}_{\mathrm{DOX}}$ for $96 \mathrm{~h}$ showed a pronounced effect, showcasing a reduction in signal of $62.62 \pm 2.05 \%(\mathrm{P}=0.002)$. All data were statistically significant compared to controls (non-treated cells), as shown by One-way ANOVA analysis. Moreover, Two-ways ANOVA statistical analysis showed a significant difference between $\operatorname{IONP}_{\mathrm{CO}}$ and $\mathrm{IONP}_{\mathrm{DOX}}(\mathrm{P}<0.0001$ for $48 \mathrm{~h} ; \mathrm{P}<0.0001$ for $72 \mathrm{~h}$; $\mathrm{P}<0.0001$ for $96 \mathrm{~h})$. Also, the presence of DOX in the construct induced a significant cytotoxic effect in the HeLa cells $(\mathrm{P}=0.0003$ for $48 \mathrm{~h} ; \mathrm{P}<0.0001$ for $72 \mathrm{~h} ; \mathrm{P}<0.0001$ for $96 \mathrm{~h})$.

Incubation with IONP for $16 \mathrm{~h}$ caused a change in the cell cycle distribution (Fig. 5a-c), evidenced through an increase in the number of cells in $\mathrm{G}_{2}$ phase, compared to non-treated controls (Fig. 5c). This effect was observed at the time of removing the NP $\left(25.90 \pm 1.90\right.$ for $\mathrm{IONP}_{\mathrm{CO}}, 26.95 \pm 1.45$ for IONP ${ }_{\mathrm{DOX}}$, respectively $18.03 \pm 6.15$ for control cells, where $\mathrm{P}=0.05$ for $\mathrm{IONP}_{\mathrm{CO}}, \mathrm{P}=0.03$ for $\mathrm{IONP}_{\mathrm{DOX}}$, compared to control), and again at $16 \mathrm{~h}$ after removing the NP $\left(21.00 \pm 4.10 \%\right.$ for $\mathrm{IONP}_{\mathrm{CO}}, 19.10 \pm 0.60 \%$ for $\mathrm{IONP}_{\mathrm{DOX}}$ and $12.90 \pm 0.70 \%$ for untreated controls; $\mathrm{P}=0.013$ for $\mathrm{IONP}_{\mathrm{CO}}$, respectively $\mathrm{P}<0.001$ for $\mathrm{IONP}_{\mathrm{DOX}}$ compared to control). The $\mathrm{G}_{2} / \mathrm{M}$ fraction decreased at $24 \mathrm{~h}$ which was paralleled by an increase of the fraction of $\mathrm{G}_{1}$ cells, suggesting induction and release of a temporary $\mathrm{G}_{2} / \mathrm{M}$ block. Measurements of cell division (number of cell doublings) (Fig. $5 \mathrm{~d}$ ) showed a minimal but statistically significant difference only at $16 \mathrm{~h}$ after nanoparticles removal caused by NPs (untreated controls vs $\mathrm{IONP}_{\mathrm{CO}}: \mathrm{P}=0.04$; untreated controls vs $\mathrm{IONP}_{\mathrm{DOX}}: \mathrm{P}=0.03$ ) as the effect was independent of DOX loading $\left(\right.$ IONP $_{\mathrm{CO}}$ Vs IONP $\left.\mathrm{IOx}_{\mathrm{DO}} \mathrm{NS}\right)$.

The clonogenic survival assay (Fig. 4d) emphasized the biocompatible character of the DOX-free NP with $\mathrm{SF}\left(\mathrm{IONP}_{\mathrm{CO}}\right)=1.07 \pm 0.38$, while the incorporation of DOX in the IONP polymeric shell caused a reduction in HeLa cells survival with $\mathrm{SF}\left(\mathrm{IONP}_{\mathrm{DOX}}\right)=0.56 \pm 0.14$ which was attributed to the release of DOX in the cells.

\section{Discussion}

In this study we designed and synthesized polyethylene glycol (PEG)-functionalized iron oxide nanoparticles $\left(\mathrm{IONP}_{\mathrm{CO}}\right)$ for the encapsulation of the chemotherapeutic substance doxorubicin (IONP $\left.\mathrm{DOX}_{\mathrm{D}}\right)$. We determined the uptake efficacy of the IONP and evaluated their ability to induce cell death after DOX loading. Results showed that most of the loaded drug was released from IONP ${ }_{\text {DOX }}$ within $24 \mathrm{~h}$, with a complete release at $70 \mathrm{~h}$. Internalization of IONP ${ }_{\mathrm{DOX}}$ and $\mathrm{IONP}_{\mathrm{CO}}$ into HeLa cells occurred by pino- and endocytosis, with both IONPs accumulating in the peri-nuclear area. DOX-free nanoparticles proved to be biocompatible for HeLa cells, while the cells treatment with $\mathrm{IONP}_{\mathrm{DOx}}$ determined a concentration and time-dependent decrease of cells proliferation.

The investigations highlight the intracellular fate of IONP after discontinuing the NP exposure, both quantitatively (through accurate measurements of the retained $\mathrm{Fe}_{3} \mathrm{O}_{4}$ per cell) and qualitatively (through electron 
microscopy imaging, in relation to the cellular compartments). Moreover, the study evaluates the IONP influence on the cell cycle and long-term proliferation/clonogenic survival after discontinuing the NP exposure.

IONP $_{\text {DOX }}$ were made using a three-step synthesis method. The novel method that we have developed is adequate for large scale extension due the advantages like ease, low costs, high yield synthesis and reproducibility. In a first step, bare $\mathrm{Fe}_{3} \mathrm{O}_{4}$ nanoparticles were produced by modified room temperature chemical co-precipitation similar to ${ }^{31}$, resulting in highly crystalline face-centred spinel structured magnetite nanoparticles ${ }^{32,33}$ (Fig. 1d). Post-synthesis conjugation of the iron oxide cores using PEG (molecular weight $6 \mathrm{KDa}$ ) resulted in individual coverage of the IONP with a low crystalline organic phase, forming core-shell-like nano-constructs with high stability and positive surface charge (Fig. 1a-d). Previous results showed that PEG-conjugated nanoparticles have a positive charge in solutions with $\mathrm{pH}<8^{34}$, in concordance with our measurements. Dissociation of $\mathrm{DOX} \cdot \mathrm{HCl}$ in water not only determined a change of IONP charge due to alteration of ions concentration in loading solution, but also led to higher hydrodynamic diameters, following DOX entrapment and interaction with PEG shells.

A challenge in developing nanoparticle-based drug delivery systems is finding an optimal design that enables internalizing, as well as retention in targeted cells. The microscopy investigations emphasized the nanoparticles localization in the cytoplasm of the cells (Fig. 2). In addition, a slight gradient effect of DOX, which might be a result of drug release from the IONP was observed (Fig. 2e and Supplementary Fig. S3). These results correlate with the DOX release data, considering the fact that the fluorescence microscopy investigations were done at $24 \mathrm{~h}$ after the end of NP treatment. Moreover, Fig. 2e proves the stability of DOX loading in IONP and suggests that the release is only triggered by the intracellular environment. In case of free DOX (Supplementary Fig. S3), due to its small size, directly diffused into the nucleus of the cell after few hours of incubation, while IONP ${ }_{\mathrm{DOx}}$ samples were showing signal mainly in the cytoplasm and weak signal in the nuclei (Supplementary Fig. S3). Instability of DOX loading in IONP in stock solutions would cause a release of DOX in the buffer solution and thus the direct diffusion of the chemotherapeutic molecule in the cell nucleus accompanied by a lack of fluorescence signal from the cytoplasmic compartment.

Similar results were reported by Zhang Y. et al. ${ }^{35}$ for $180 \mathrm{~nm}$ Poly(ethylene glycol)- doxorubicin-curcumin nanoparticles which were mainly located in the vicinity of the nucleus at low incubation times, while the free drug diffused into the nucleus of the cells, due to a differentiation in the uptake pathways. This changed with time, as the active substances were released from the constructs.

For all experimental conditions, agglomerates of IONP could be observed both in the peri-nuclear area and the cytoplasm (Figs. 2, 3). The presence of IONP $_{\mathrm{CO}}$ was mostly observed in vesicle-like structures (Fig. 3a,b), due to entrapment in the endo-/lysosomal compartments ${ }^{36}$, while IONP $\mathrm{DOX}_{\mathrm{D}}$ were also found in the cytoplasm (Fig. 3c,d). Bypassing the endo-/lysosomal system is a requirement in the design of intracellular drug delivery systems ${ }^{37}$.

Petros et al..$^{38}$ stated that nanoparticles having higher hydrodynamic diameter are transported across the cellular membrane via clathrin-mediated endocytosis or macropinocytosis. This was confirmed by Pearson et al. ${ }^{39}$ and Jana ${ }^{40}$. Bannunah et al. ${ }^{41}$ showed that dimension does not play an important role in the NP mechanism of internalizing, but it is dependent on surface charge; also, there is more than one mechanism involved in the internalizing of the same type of NP. Our results show that both types of constructs are internalized via macropinocytosis (Fig. 3e and Supplementary Fig. S4c) and sometimes smaller aggregates are internalized via endocytosis (Fig. 3f and Supplementary Fig. S4d). Both mechanisms were observed for DOX-loaded and DOX-free IONP, meaning that the cells do not use one mechanism or another based on surface charge, but rather on dimension. Extensions of the cellular membrane surround the NP agglomerates in the vicinity of the membrane, forming micrometre-sized vesicles and getting the constructs into the intracellular compartment. Eventually, these get trapped in lysosomes for cellular disposal (Fig. $3 \mathrm{~g}$ and Supplementary Fig. S4e).

Our observations agree with results from other publications ${ }^{26}$. However, in case of DOX-loaded constructs, the major fraction of NP seem to escape the lysosomal trapping and to be freely located into the cytoplasm at $24 \mathrm{~h}$ after discontinuing the exposure of HeLa cells to IONP $_{\text {DOX }}$ (Fig. 3c,d). The development and fate of PEG conjugated iron oxide nanoparticles to escape the endo-/ lysosomal trapping in the context of drug delivery is not well studied. This bypassing approach of iron oxide nanoparticles has been investigated for the purpose of radiosensitization, in case of dextran coated iron oxide nanoparticles conjugated with a cell penetrating peptide $(\mathrm{TAT})^{42}$ and citrate coated superparamagnetic iron oxide nanoparticles ${ }^{43}$.

Lysosome function is to digest the internalized material taken up by the cell by means of endocytosis ${ }^{44}$, thus escaping or bypassing the lysosome uptake might be a solution to improve organelle specific targeting. In case of nanoparticle formulas, endosome and lysosome inclusions might also be an obstacle for effective treatment, as stated by Lloyd $\mathrm{d}^{45}$. Whereas nano-carrier degradation by lysosome microenvironment and liberation of the active substance may still be considered one important principle of nanoparticle-based drug delivery systems, lysosome membrane can act as a natural barrier against efficient drug release ${ }^{46}$. In this case, cytosolic delivery of the drugs by nanoparticles escaping lysosomal entrapment ${ }^{47,48}$ might be a key to successful killing of the cancer cells.

Besides efficient uptake of nanoparticles in cancer cells, their retention is also important so that an effective high concentration can be reached. While small nanoparticles (diameters lower than $50 \mathrm{~nm}$ ) undergo exocytosis within $24 \mathrm{~h}$ of uptake, larger nanoparticles or aggregates are retained for longer periods of time ${ }^{49}$. Our microscopy investigations emphasized the presence of IONP after $40 \mathrm{~h}$ ( $16 \mathrm{~h}$ of incubation with NPs and additional $24 \mathrm{~h}$ incubation without NPs), while quantitative measurements showed that, at this time-point, a 3.6-fold higher concentration of $\mathrm{Fe}_{3} \mathrm{O}_{4}$ was measured in $\mathrm{IONP}_{\text {DOx }}$ compared to IONP $\mathrm{CO}$ (Fig. 3h). This difference in internalized $\mathrm{Fe}_{3} \mathrm{O}_{4}$ in the two groups of IONP-exposed HeLa cells might be due to the difference in hydrodynamic diameter (almost double for IONP $\mathrm{DOX}_{\mathrm{DOX}}$ compared to $\mathrm{IONP}_{\mathrm{CO}}$ ), but also due to the induction of cell death in case of DOX-loaded nanoparticles, which can artificially increase the concentration $\mathrm{Fe}_{3} \mathrm{O}_{4} /$ cell. To the best of our knowledge this is the first study to evaluate the intracellular retaining and fate of PEG-coated iron oxide nanoparticles (qualitatively, 
in relation to the cellular compartments and also quantitatively, by providing an accurate concentration of NP per individual cell) at periods of time longer than one complete cell cycle, after the termination of NP exposure.

Considering compatibility to human blood, our results showed biocompatibility for both $\mathrm{IONP}_{\mathrm{CO}}$ and $\mathrm{IONP}_{\text {DOx }}$ (Supplementary Material Section 7), matching data from the literature ${ }^{50-52}$. Concerning the cytotoxicity and proliferation exhibited by HeLa cells after IONP exposure, our data (Fig. 4a-c) showed that, in the first days of interaction between cells and PEG-functionalized NP, a weak cytotoxic effect occurred already at very small concentrations, which did not increase with concentration, after a certain threshold, the amount of cytotoxicity being maintained almost constant. Similar results were obtained by Xia et al. ${ }^{53}$ for a redox responsive polyethylene glycol- $\mathrm{Fe}_{3} \mathrm{O}_{4}$ nanoparticles self-assembled micelles. However, at later time points $(96 \mathrm{~h})$, the cell proliferation was maintained above $80 \%$ limit (relative to control), which is a threshold for biocompatibility (ISO 10993-12:2001(E) ${ }^{54}$ ). Long-term monitoring (Fig. 4d) showed that IONP $_{\mathrm{CO}}$ did not affect the clonogenic survival of HeLa cells after 14 days. Other studies also reported PEG-coated iron oxide nanoparticles as biocompatible ${ }^{55-57}$. Feng et al. ${ }^{26}$ showed that such nanoparticles could induce autophagy in vitro, but otherwise showed no obvious signs of in vivo toxicity in BALB/c mice.

Results for IONP $\mathrm{DOX}_{\text {DOX }}$ showed that DOX-containing NP caused significant cytotoxicity and growth inhibition in HeLa cells compared to untreated control samples (Fig. 4a-c). This effect was concentration and time dependent, showing that these constructs can be efficiently used to induce cell death in human cervical adenocarcinoma cells. The lack of recovery at $96 \mathrm{~h}$ (as compared to $\mathrm{IONP}_{\mathrm{CO}}$ ) suggests that $\mathrm{IONP}_{\mathrm{DOX}}$ not only caused growth delay but also real cytotoxicity. The clonogenic survival data confirmed this by showing a reduced SF in cells treated with $\mathrm{IONP}_{\mathrm{DOX}}$ after 14 days compared to $\mathrm{IONP}_{\mathrm{CO}}$-treated cells (Fig. 4d).

The cell cycle arrest at $16 \mathrm{~h}$ after $\operatorname{IONP}_{\mathrm{CO}}$ treatment was also observed for $\operatorname{IONP}_{\mathrm{DOX}}$ (Fig. 5) indicating little effect of DOX released from the nanoparticles. In all, results showed no statistically significant difference in cell cycle distribution between the $\mathrm{IONP}_{\mathrm{CO}}$ and $\mathrm{IONP}_{\mathrm{DOx}}$ groups (Fig. 5).

This study shows the generation and characterization of polyethylene glycol-functionalized iron oxide nanoparticle that have shown efficient internalizing and retention in human cervical adenocarcinoma cells. Highly crystalline, bio- and hemocompatible nanoparticles, IONP have the ability to encapsulate and deliver the chemotherapeutic doxorubicin directly into the intracellular cytoplasmic compartment and thereby efficiently causing cell death in the cells. This makes them potential candidates for nanoparticle-mediated and chemotherapy-induced inactivation of tumour cells.

\section{Methods}

Synthesis and characterization of the IONP. The synthesis of the IONP was done in 3 steps (described in more detail in the Supplementary Material Section 1): (1) Synthesis of bare iron oxide nanoparticles through chemical co-precipitation, (2) post-synthesis functionalizing of iron oxide nanoparticles with polyethylene glycol (PEG) $6000 \mathrm{Da}\left(\mathrm{IONP}_{\mathrm{CO}}\right.$ ) and (3) optional loading of the $\mathrm{IONP}_{\mathrm{CO}}$ with doxorubicin (only in IONP $\mathrm{DOx}_{\text {). }}$ ).

The morphology, crystallinity and mineralogical composition of the IONP was examined using transmission electron microscopy (TEM) on a Tecnai G2 F30 S-TWIN HR-TEM (Thermo Fisher Scientific, Hillsboro, OR, USA) equipment with selected area electron diffraction (SAED) module, for which sample preparation has been described previously ${ }^{31}$. The analysis of the loading efficiency of DOX into the nano-constructs is described in Supplementary Material Section 2.

The hydrodynamic diameter and surface charge (zeta potential) of IONP were characterized using a Delsa Nano C instrument (Beckman Coulter, Brea, CA, USA) and recorded using the DelsaNano 3.73 software (Beckman Coulter). The measurements were done for freshly prepared nanoparticle suspensions in ultrapure water without prior ultrasound dispersion, based on the existing international documentary standards ISO 13321:1996 $6^{58}$ and ISO 22412: $2008 b^{59}$.

The release kinetics of DOX from IONP $_{\mathrm{DOX}}$ was measured for media with different biologically relevant $\mathrm{pH}$ : 7.4, 6.5 and 4.7. Studies were done in standard conditions of temperature and humidity $\left(37 \pm 2{ }^{\circ} \mathrm{C}, 5 \pm 1 \% \mathrm{CO}_{2}\right)$. Samples were prepared and analysed as described in the Supplementary Material Section 3 and performed once in triplicate.

Cell culture. The biological evaluation of the IONP was performed on the human cervical adenocarcinoma cell line HeLa, which was obtained from the Tumour Cell Bank of the German Cancer Research Centre (DKFZ, Heidelberg, Germany). This cell line was chosen because it has been previously used in a variety of studies ${ }^{49,60,61}$ evaluating novel nanoconstructs and thereby allows a comparison of results. Cells were cultured in Dulbecco's modified Eagle's medium (DMEM) (Biochrom, Merck KGaA, Darmstadt, Germany) supplemented with $10 \%$ FBS (Biochrom, Merck KGaA). Cell cultures were maintained at $37^{\circ} \mathrm{C}$ in a humidified incubator $\left(95 \%\right.$ air, $\left.5 \% \mathrm{CO}_{2}\right)$.

Treatment and incubation with IONP. HeLa cells were seeded in appropriate concentration for each investigation and allowed to attach for $4 \mathrm{~h}$. Afterwards, the culture medium was replaced with fresh medium containing nanoparticles in the respective concentrations and incubated for $16 \mathrm{~h}$.

Uptake and retention of IONP. The uptake and retention of IONP by HeLa cells was evaluated by three microscopic methods generating complementary information on the localizing of the nanoparticles in relation to the cultured tumour cells. The preparation of the samples for microscopy is described in the Supplementary Material Section 4. Optical visualization of IONP in HeLa cells $24 \mathrm{~h}$ after incubation (for $16 \mathrm{~h}$ ) in presence of nanoparticles was performed using a Prussian Blue staining, resulting in a light blue colouring of sub-micron structures. Fluorescence imaging was possible due to native property of doxorubicin. Optical and fluorescence microscopy were performed using a Leica DMRE microscopy equipped with a Leica DFC3000G camera (Leica Mikrosysteme Vertrieb GmbH Mikroskopie und Histologie, Wetzlar, Germany) and an Axio Observer Z1 
microscope (ZEISS, Oberkochen, Germany) equipped with an Axiocam 506 camera. Images were acquired using ZEN 2 software (ZEISS). Samples for transmission electron imaging were prepared similarly as for optical and fluorescence microscopy imaging (as described in Supplementary Material Section 4). Images were acquired using a Zeiss EM 10 transmission electron microscope (ZEISS) equipped with an Olympus Megaview G2 camera (Olympus Europa SE \& Co. KG, Hamburg, Germany).

Particle-induced X-Ray emission (PIXE) was performed using a 3 MV Tandetron accelerator with a $2.7 \mathrm{MeV}$ proton beam and an in-air irradiation setup ${ }^{62}$. The characteristic X-Rays were recorded by an Amptek silicon drift detector (SDD) positioned at $45^{\circ}$ with respect to the beam direction and the spectra were processed with the GUPIXWIN 2.2.4 software ${ }^{63}$. The SDD resolution is $130 \mathrm{eV}$ at $5.9 \mathrm{keV}\left(\mathrm{K}_{\alpha}\right.$ line of $\left.{ }^{55} \mathrm{Mn}\right)$. The concentration of $\mathrm{Fe}_{3} \mathrm{O}_{4}$ per cell was calculated by normalizing the output values to the viable cell number at each corresponding time point.

Proliferation and clonogenic survival. The quantitative effects of the IONP on proliferation kinetics were evaluated using a tetrazolium salt-based proliferation assay (MTT, Sigma-Aldrich Chemie GmbH, Taufkirchen, Germany). Dose-response curves were obtained for concentrations up to $200 \mu \mathrm{g} / \mathrm{mL}$ IONP continuous exposure for 48, 72 and $96 \mathrm{~h}$. Samples were prepared as described in the Supplementary Material Section 5.

Cells for longer term survival evaluation (14 days) were incubated with IONP for $16 \mathrm{~h}$ and then detached and reseeded into the colony formation assay at 200 cells $/ 25 \mathrm{~cm}^{2}$ flask as described in Supplementary Material Section 5 .

The surviving fraction $(\mathrm{SF})$ was fitted with the linear-quadratic model $\left(\ln (\mathrm{SF})=-\left(\alpha \mathrm{D}+\beta \mathrm{D}^{2}\right)\right)$ using the non-linear regression tool of SigmaPlot 12 (Systat Software GmbH, Erkrath, Germany) ${ }^{49}$.

Cell cycle distribution and doubling time. The change in cell cycle distribution and division of HeLa cells exposed during $16 \mathrm{~h}$ with IONP was evaluated for cells treated and stained as described in Supplementary Material Section 6. Acquisition was performed on the BD FACSLyric (BD Biosciences, San Jose, CA, USA), and analysed using FlowJo 10.5 software (BD Biosciences).

Statistical analysis. All data are presented as mean \pm SEM from three independent experiments, unless specified otherwise. Statistical analysis was done using t-test, one-way ANOVA (SigmaPlot 12) and two-way ANOVA (Prism 5, GraphPad, San Diego, USA).

\section{Data availability}

All data generated or analysed during this study are included in this published article (and its Supplementary Information File).

Received: 25 February 2020; Accepted: 1 June 2020;

Published online: 29 June 2020

\section{References}

1. Shi, J., Kantoff, P. W., Wooster, R. \& Farokhzad, O. C. Cancer nanomedicine: progress, challenges and opportunities. Nat. Rev. Cancer. 17(1), 20-37 (2016).

2. Greish, K. Enhanced Permeability and Retention (EPR) Effect for Anticancer Nanomedicine Drug Targeting in Cancer Nanotechnology. Methods in Molecular Biology (Methods and Protocols) (ed. Grobmyer, S. \& Moudgil, B.) 624, 25-37 (Humana Press, 2010).

3. Russell, L. M., Dawidczyk, C. M. \& Searson, P. C. Quantitative Evaluation of the Enhanced Permeability and Retention (EPR) Effect in Cancer Nanotechnology. Methods in Molecular Biology (ed. Zeineldin, R.) 1530, 247-254 (Humana Press, 2017).

4. Nakamura, Y., Mochida, A., Choyke, P. L. \& Kobayashi, H. Nanodrug Delivery: Is the Enhanced Permeability and Retention Effect Sufficient for Curing Cancer? Bioconj. Chem. 27(10), 2225-2238 (2016).

5. Wu, M. \& Huang, S. Magnetic nanoparticles in cancer diagnosis, drug delivery and treatment. Mol. Clin. Oncol. 7(5), 738-746 (2017).

6. Li, Z., Zhang, J., Guo, X., Guo, X. \& Zhang, Z. Multi-functional magnetic nanoparticles as an effective drug carrier for the controlled anti-tumor treatment. J. Biomater. Appl. 32(7), 967-976 (2018).

7. Ali, A. A. A. et al. Erlotinib-Conjugated Iron Oxide Nanoparticles as a Smart Cancer-Targeted Theranostic Probe for MRI. Sci. Rep. 6, 36650 (2016).

8. Qiu, Y. et al. Magnetic forces enable controlled drug delivery by disrupting endothelial cell-cell junctions. Nat. Commun. 8, 15594 (2017).

9. Bijaideep, D. et al. $\mathrm{pH}$ sensitive surfactant-stabilized Fe3O4 magnetic nanocarriers for dual drug delivery. Colloids Surf. $B$ Biointerfaces. 162, 163-171 (2018).

10. Himani, K., Rajput, S., Kumar, B. N. P., Mandal, M. \& Pathak, A. $\mathrm{Fe}_{3} \mathrm{O}_{4} @ z$ zirconium phosphate core-shell nanoparticles for pHsensitive and magnetically guided drug delivery applications. RSC Adv. 6, 21285-21292 (2016).

11. Moorthy, M. S. et al. Synthesis of Fe3O4 modified mesoporous silica hybrid for $\mathrm{pH}$-responsive drug delivery and magnetic hyperthermia applications. J. Porous Mat. 25(4), 1251-1264 (2017).

12. Yang, Z. et al. pH-Sensitive Composite with Controlled Multistage Drug Release for Synergetic Photothermal Therapy and Chemotherapy. Eur. J. Inorg. Chem. 2017(47), 5536-5628 (2017).

13. Dani, R. K., Schumann, C. \& Taratula, O. Temperature-tunable iron oxide nanoparticles for remote-controlled drug release. AAPS. Pharm. Sci. Tech. 15(4), 963-72 (2014).

14. Iglesias, G. R., Reyes-Ortega, F., Fernandez, B. L. C. \& Delgado, A. V. Hyperthermia-Triggered Gemcitabine Release from PolymerCoated Magnetite Nanoparticles. Polym. 10(3), 269 (2018).

15. Chen, L., Wu, L., Liu, F., Qi, X. Y. \& Shen, S. Azo-functionalized Fe3O4 nanoparticles: a near-infrared light triggered drug delivery system for combined therapy of cancer with low toxicity. J. Mater. Chem. B. 4, 3660-3669 (2016).

16. Estelrich, J. \& Busquets, M. A. Iron Oxide Nanoparticles in Photothermal Therapy. Molecules. 23(7), 1567 (2018).

17. Neel, N. et al. Radiofrequency-Triggered Drug Release from Nanoliposomes with Millimeter-Scale Resolution Using a Superimposed Static Gating Field. Small 14(44), e1802563 (2018).

18. Bozuyuk, U. et al. Light-Triggered Drug Release from 3D-Printed Magnetic Chitosan Microswimmers. ACS Nano. 12(9), 9617-9625 (2018). 
19. Chen, C. W. et al. Encapsulation of $\mathrm{Au} / \mathrm{Fe}_{3} \mathrm{O}_{4}$ nanoparticles into a polymer nanoarchitecture with combined near infrared-triggered chemo-photothermal therapy based on intracellular secondary protein understanding. J. Mater. Chem. B. 5, 5774-5782 (2017).

20. Liu, J. et al. $\mathrm{Fe}_{3} \mathrm{O}_{4}$-based PLGA nanoparticles as MR contrast agents for the detection of thrombosis. Int. J. Nanomed. 12, 1113-1126 (2017).

21. Ghorbaanee, M., Salarian, A. A. \& Saba, V. Curcumin Loaded $\mathrm{Fe}_{3} \mathrm{O}_{4}$ Nanoparticles: An MRI Contrast Agent to Investigate the Impact of Curcumin on Maximizing Negative Contrast and $\mathrm{r}_{2}$ Relaxation Rate. J. Inorg. Organomet. Polym. Mat. 28(5), 2169-2178 (2018).

22. Shen, Z., Wu, A. \& Chen, X. Iron Oxide Nanoparticle Based Contrast Agents for Magnetic Resonance Imaging. Mol. Pharm. 14(5), $1352-1364$ (2017).

23. https://www.magforce.com/home.html (2019).

24. Tudisco, C. et al. Comparison Between Folic Acid and gH625 Peptide-Based Functionalization of Fe3O4 Magnetic Nanoparticles for Enhanced Cell Internalization. Nanoscale Res. Lett. 13(1), 45 (2018).

25. Chaves, N. L. et al. Exploring cellular uptake of iron oxide nanoparticles associated with rhodium citrate in breast cancer cells. Int. J. Nanomed. 12, 5511-5523 (2017).

26. Feng, Q. et al. Uptake, distribution, clearance, and toxicity of iron oxide nanoparticles with different sizes and coatings. Sci. Rep. 8 , 2082 (2018).

27. van Sluis, R. et al. In vivo imaging of extracellular $\mathrm{pH}$ using ${ }^{1} \mathrm{H}$ MRSI. Magn. Reson. Med. 41, 743-750 (1999).

28. Estrella, V. et al. Acidity generated by the tumor microenvironment drives local invasion. Cancer Res. 73(5), 1524-35 (2013).

29. Mindell, J. A. Lysosomal Acidification Mechanisms. Annu. Rev. Physiol. 74(1), 69-86 (2012).

30. Kollmorgen, G. M. \& Griffin, M. J. The effect of hydrocortisone on HeLa cell growth. Cell Tissue Kinet. 2(2), 111-122 (1969).

31. Popescu, R. C. et al. Fabrication and Cytotoxicity of Gemcitabine-Functionalized Magnetite Nanoparticles. Molecules. 22, 1080 (2017).

32. Araújo-Neto, R. P. et al. Monodisperse sodium oleate coated magnetite high susceptibility nanoparticles for hyperthermia applications. J. Magn. Magn. Mat. 364, 72-79 (2014).

33. Thapa, B., Diaz- Diestra, D., Beltran-Huarac, J., Weiner, B. R. \& Morell, G. Enhanced MRI T 2 relaxivity in Contrast- Probed AnchorFree PEGylated Iron Oxide Nanoparticles. Nanoscale Res. Lett. 12, 312 (2017).

34. Bloemen, M. et al. Improved functionalization of oleic acid-coated iron oxide nanoparticles for biomedical applications. J Nanopart. Res. 14(9), 1100 (2012).

35. Zhang, Y. et al. Co-delivery of doxorubicin and curcumin by $\mathrm{pH}$-sensitive prodrug nanoparticles for combination therapy of cancer. Sci Rep. 6, 21225 (2016).

36. Kamba, S. A., Ismail, M., Hussein-Al-Ali, S. H., Ibrahim, T. A. \& Zakaria, Z. A. In vitro Delivery and Controlled Release of Doxorubicin for Targeting Osteosarcoma Bone Cancer. Molecules. 18, 10580-10598 (2013).

37. Rosenblum, D. et al. Progress and challenges towards targeted delivery of cancer therapeutics. Nat Commun 9, 1410 (2018).

38. Petros, R. A. \& DeSimone, J. M. Strategies in the design of nanoparticles for therapeutic applications. Nat. Rev. Drug Discovery. 9 , 615-627 (2010).

39. Pearson, R. M., Hsu, H., Bugno, J. \& Hong, S. Understanding nano-bio interactions to improve nanocarriers for drug delivery. MRSBulletin. 39(3), 227-237 (2014)

40. Jana, N. R. Design and development of quantum dots and other nanoparticles based cellular imaging probe. Phys. Chem. Chem. Phys. 13, 385-396 (2011).

41. Bannunah, A. M., Vllasaliu, D., Lord, J. \& Stolnik, S. Mechanisms of Nanoparticle internalization and transport across and intestinal epithelial cell model: effect of size and surface charge. Mol. Pharma. 11(12), 4363-4373 (2014).

42. Hauser, A. K. et al. Targeted iron oxide nanoparticles for the enhancement of radiation therapy. Biomaterials. 105, 127-135 (2016).

43. Klein, S. et al. Superparamagentic iron oxide nanoparticles as novel X-ray enhancer for low-dose radiation therapy. J Phys Chem B. 118(23), 6159-6166 (2014).

44. Cooper, G. M. Chapter 9. Protein Sorting and Transport- The Endoplasmic Reticulum, Golgi Apparatus, and Lysosomes in The Cell: A Molecular Approach. (Sunderland: Sinauer Associates, 2000).

45. Lloydd, J. B. The lysosome/endosome membrane: a barrier to polymer-based drug delivery? Macromol Symposia. 172(1), 29-34 (2001).

46. Castellani, K. C., Perry, E. A., Perry, G., Castellani, R. J. \& Treating Alzheimer Disease: Is Diet and Exercise more Effective than Small Molecule Therapy? J. Membra. Sci. Technol. 3, el11 (2016).

47. Agardan, N. B. M. \& Torchilin, V. P. Chapter 1- Engineering of stimuli- sensitive nanopreparations to overcome physiological barriers and cancer multidrug resistance in Engineering of Nanobiomaterials: Applications of Nanobiomaterials (ed. Grumezescu A), 2, 1-28 (William Andrew, 2016).

48. Hillaireau, H. \& Couvreur, P. Nanocarriers' entry into the cell: relevance to drug delivery. Cell Mol. Life Sci. 66(17), 2873-96 (2009).

49. Burger, N. et al. A method for the efficient cellular uptake and retention of small modified gold nanoparticles for the radiosensitization of cells. Nanomed NBM. 10(6), 1365-73 (2014).

50. Ma, D., Chen, J., Luo, Y., Wang, H. \& Shi, X. Zwitterion-coated ultrasmall iron oxide nanoparticles for enhanced $\mathrm{T}_{1}$-weighted magnetic resonance imaging applications. J. Mater. Chem. B. 5, 7267-7273 (2017).

51. Xue, W. et al. Effects of core size and PEG coating layer of iron oxide nanoparticles on the distribution and metabolism in mice. Int. J. Nanomed. 13, 5719-5731 (2018).

52. Ali, L. M. et al. Hemostasis disorders caused by polymer coated iron oxide nanoparticles. J Biomed Nanotechnol 9(7), 1272-85 (2013).

53. Xia, K. et al. Nanocarriers of $\mathrm{Fe}_{3} \mathrm{O}_{4}$ as a Novel Method for Delivery of the Antineoplastic Agent Doxorubicin Into HeLa Cells in vitro. Front Oncol. 9, 250 (2019).

54. International Organization for Standardization, ISO 10993-12:2001(E)- Biological evaluation of medical devices, Part 12: Sample preparation and reference materials, https://www.iso.org/standard/53468.html (2020).

55. Sangeetha, J., Susha, T., Arutchelvi, J., Mukesh, D. \& John, P. Functionalization of Iron Oxide Nanoparticles with Biosurfactants and Biocompatibility Studies. J. Biomed. Nanotechnol. 9(5), 751-764(14) (2013).

56. Yuan, G., Yuan, Y., Xu, K. \& Luo, Q. Biocompatible PEGylated Fe3O4 Nanoparticles as Photothermal Agents for Near-Infrared Light Modulated Cancer Therapy. Int. J. Mol. Sci. 15(10), 18776-18788 (2014).

57. Kansara, K. et al. Synthesis of biocompatible iron oxide nanoparticles as a drug delivery vehicle. Int. J. Nanomed. 13(T-NANO 2014 Abstracts), 79-82 (2018).

58. International Organization for Standardization, ISO 13321:1996- Particle size analysis- Photon correlation spectroscopy, https:// www.iso.org/standard/21707.html (2020).

59. International Organization for Standardization, ISO 22412:2008- Particle size analysis- Dynamic light scattering (DLS), https:// www.iso.org/standard/40942.html (2020).

60. Yang, Y. et al. Promising Nanocarriers for PEDF Gene Targeting Delivery to Cervical Cancer Cells Mediated by the Over-expressing FRo. Sci. Rep. 6, 32427 (2016).

61. Liu, Y. et al. Dynamically-enhanced retention of gold nanoclusters in HeLa cells following X-rays exposure: A cell cycle phasedependent targeting approach. Radiother. Oncol. 119(3), 544-551 (2016). 
62. Burducea, I. et al. A new ion beam facility based on a 3 MV Tandetron at IFIN-HH. Romania. Nuclear Inst. Met. Phys. Res. B. 359, $12-19(2015)$

63. Campbell, J. L., Boyd, N. I., Grassi, N., Bonnick, P. \& Maxwell, J. A. The Guelph PIXE software package IV. Nuclear Inst. Met. Phys. Res. B. 268(20), 3356-3363 (2010).

\section{Acknowledgements}

We gratefully acknowledge Miriam Bierbaum and Adriana Grbenicek for excellent technical assistance. The synthesis and characterization of the nanoparticles was done at "Horia Hulubei" National Institute for Physics and Nuclear Engineering in collaboration with Politehnica University of Bucharest, work supported by Romanian Ministry of Research National [grants no. PN 18090202 and 64PCCDI/2017]. The structural and compositional characterizing of the materials through transmission electron microscopy was possible due to an EU-funded project [POSCCE-A2-O2.2.1-2013-1/Priority Axe 2, Project no 638/12.03.2014, ID 1970, SMIS-CSNR code 48652]. The in vitro evaluation of the nanoparticles was performed at the Universitätsmedizin Mannheim, Medical Faculty Mannheim, Heidelberg University, for which Roxana C. Popescu declares financial support from the Deutscher Akademischer Austauschdienst (DAAD) [grant no. 57299291].

\section{Author contributions}

R.C.P., D.S., I.D., C.H. and M.R.V. conceived and designed the experiments; R.C.P. and I.D. designed and synthesized the nanoparticles; B.S.V., A.B. and R.C.P. performed the characterization of the nanoparticles; R.C.P., H.H. and M.T. performed the in vitro testing and characterization; M.S. and D.A.I. performed the PIXE measurements and data processing; R.C.P., D.S., C.H. and M.R.V. analysed the data; R.C.P., D.S., C.H. and M.R.V. drafted the manuscript; R.C.P., D.S., E.A., F.W., F.A.G., C.H. and M.R.V. participated in the coordination. All authors have approved the manuscript.

\section{Competing interests}

The authors declare no competing interests.

\section{Additional information}

Supplementary information is available for this paper at https://doi.org/10.1038/s41598-020-67207-y.

Correspondence and requests for materials should be addressed to D.S. or M.R.V.

Reprints and permissions information is available at www.nature.com/reprints.

Publisher's note Springer Nature remains neutral with regard to jurisdictional claims in published maps and institutional affiliations.

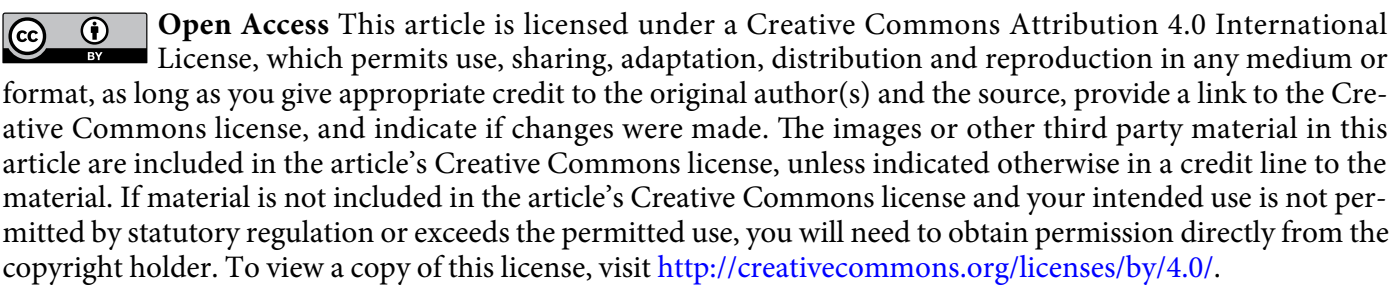

(c) The Author(s) 2020 